\title{
Genetic imprinting of IGF2/H19 in Normal, Hyperplastic and Neoplastic Cells
}

\author{
R. Ohlsson, T.J. Ekström, G. Adam, S. Miller, H. Cui, R. Fisher, \\ C. Walsh \\ Department of Animal Development \& Genetics, Uppsala University, Uppsala, Sweden
}

Genetic imprinting implies the preferential or exclusive expression of one of the parental alleles of a subset of autosomal loci. The insulin-like growth factor II (IGF2) and HI9 loci are particularly interesting examples of this phenomenon since their products appear to display growth agonistic and antagonistic properties, respectively. In addition, IGF2 and $H 19$ are only $90 \mathrm{~kb}$ apart, are expressed from opposite parental alleles $[1,2]$ and show a striking similarity in their spatial expression patterns during human prenatal development [3]. One exception is the choroid plexus and leptomeninges which express $I G F 2$ biallically with no detectable $H 19$ expression [3]. Observations like these have fuelled ideas that there is an enhancer competition between the IGF2 and $H 19$ loci [4]. The imprinting status of the $H 19$ locus would then indirectly control the expressivity of $I G F 2$. This model is likely to be too simple since the $\mathrm{P} 1$ promoter of $I G F 2$ is not functionally imprinted during liver development in humans [4]. Moreover, while the liver P2-P4 promoters are expressed primarily from the paternally derived allele during human prenatal development, the $\mathrm{P} 2-\mathrm{P} 4$ promoters can be expressed from both parental alleles in complex patterns during postnatal human development [5]. The enhancer competition model might be put to the test in human and mouse uniparental embryos since the parental origin of their diploid genomes cannot be discerned. Unexpectedly, H19 which is expressed preferentially from the maternal allele in mouse [6] and human [7] placenta is expressed in both mouse and human trophoblasts (in complete hydatidiform moles) lacking the maternal genome. In the normal human placenta, the repressed paternal $H 19$ allele is more methylated. Interestingly, the $\mathrm{CpG}$ methylation pattern of $H 19$ is strikingly similar between normal placenta and complete moles. Hence, both paternal $H 19$ alleles are similarly methylated indicating that postzygotic modification events typical of normal development have taken place in complete moles as well in spite of the absence of the maternal genome. In contrast to the normal placenta, H19 is expressed biallelically in complete moles as assessed by allele-specific in situ hybridisation analysis of dispermic moles [8]. We discuss these results in relation to current models of IGF2/HI9 imprinting mechanism(s). 


\section{R. Ohlsson et al.}

\section{REFERENCES}

1. Ohlsson et al., Nature Genet 4, 94-97 (1993).

2. Rainier et al., Nature 362, 747-749 (1993).

3. Ohlsson et al., Development 120, 361-368 (1994).

4. Bartolomei, et al., Genes \& Develop 7, 1663-1673 (1993).

5. Ekstrom and Ohlsson, submitted for publication.

6. Walsh et al., Mech Develop 46, 55-62 (1994).

7. Walsh et al., submitted for publication.

8. Adam, Miller et al., manuscript.

Correspondence: Rolf Ohlsson, Department of Animal Development \& Genetics, Uppsala University, Norbyvägen 18A, S-75236, Uppsala, Sweden. 